\title{
Measurement of digital MEMS microphones
}

\author{
Michael Loibl ${ }^{1}$, Sebastian Walser ${ }^{1}$, Josef Klugbauer ${ }^{1}$, Gregor Feiertag ${ }^{1}$, Christian Siegel ${ }^{2}$ \\ ${ }^{1}$ University of Applied Sciences Munich, Lothstraße 64, 80335 Munich, Germany \\ ${ }^{2}$ EPCOS AG A TDK Group Company, Anzinger Straße 13, 81617 Munich, Germany
}

\begin{abstract}
This paper presents two methods to measure the electro-acoustic parameters of digital MEMS microphones with a pulse density modulated (PDM) output signal. The first method is based on a spectrum analysis of the PDM signal. Here a large number of data points must be recorded to achieve a good frequency resolution. With the large number of data points calculating the characteristic values takes a long time. The second method is based on an analog transformation and a subsequent decimation. With this method the same measurement quality can be reached in a fraction of the calculation time.
\end{abstract}

Keywords: digital microphone, silicon MEMS, PDM, sensitivity, signal to noise ratio

\section{Introduction}

Silicon micromachined microphones (MEMSmicrophones) are an important part of modern consumer electronic products. They are an essential part of devices like smartphones, cameras and tablets. In the last few years they have replaced electret condenser microphones because of their small size, resistance against soldering heat and a very high sound quality. $[1,2]$

Microphones transform an acoustic signal into an electrical signal. The electrical signal could be analog or digital, depending on the type of the microphone. In the last few years the marked share of digital MEMS microphones has shown a rapid increase. The main requirements for MEMS microphones are a high signal to noise ratio (SNR), small total harmonic distortions (THD) at high sound pressures and a small size. To fulfil these high demands digital MEMS microphones can be very helpful.

Because of their digital interface they are less susceptible to picking up RF interference with the interconnect lines on the board which could cause noise or distortion of the audio signal. So smartphone producers have more opportunities to place the microphones in their products for example nearer to the Wi-Fi antenna, the Bluetooth antenna or the cellular antenna. [3]

This article shows how to measure the electroacoustic characteristics of a digital MEMS microphone even without knowing internal voltage levels and only by investigation of the output signal. The measured parameters are sensitivity, SNR and THD.

\section{Basic structure of a MEMS microphone}

Most condenser silicon MEMS microphones consist of two chips. A sensor chip converts the acoustic signal into an electrical signal. The application-specific-integrated-circuit (ASIC) is necessary for signal processing and for the interface to the system. Figure 1 shows an example of a wire bonding type bottom port MEMS microphone [4].

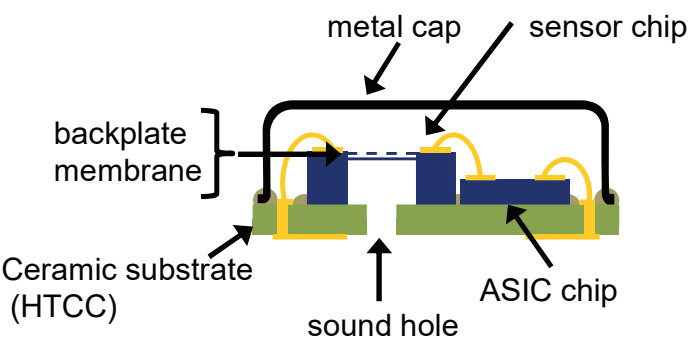

Fig. 1: Schematic cross section of a wire bonding type MEMS-microphone [4]

Other packaging technologies like flip-chip are described in [5]. According to $[6,7,8]$ a differential sensor chip with two backplates leads to a higher sensitivity and a higher SNR.

\section{Characteristics of a digital MEMS microphone}

Several parameters are used to specify digital MEMS microphones. One very important parameter is sensitivity. It is the ratio of the digital output value to the input pressure with 
the unit dBFS (decibels with respect to digital full-scale). For the measurement of sensitivity a $1 \mathrm{kHz}$ sine wave at $94 \mathrm{~dB}$ sound pressure level (SPL) or $1 \mathrm{~Pa}$ is excited in a pressure chamber.

The SNR is the ratio between the sensitivity and the noise level at the microphone output in a quiet environment. To calculate the noise level an A-weighting filter is used.

The THD is the parameter which indicates the distortion of the electrical output signal when an undistorted signal is applied. The calculating routine for a THD is shown in equation 1 .

$T H D=\frac{\sqrt{U_{2}^{2}+U_{3}^{2}+U_{4}^{2}+\ldots+U_{n}^{2}}}{U_{1}}$

In this paper we describe how these three parameters can be calculated with the two methods.

\section{Digital vs. analog MEMS microphones}

There are analog and digital microphones on the market. Differences of these two groups are the type of the electrical output signal and an optical difference. The connecting pads in bottom-view for such microphones are shown in Figure 2.
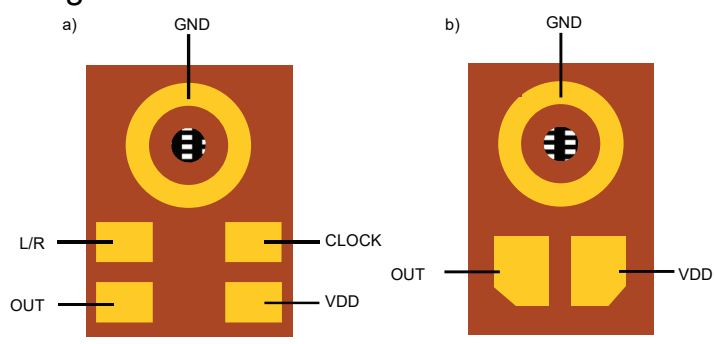

Fig. 2: Bottom-view of a) a digital microphone $\left(3.5 \times 2.65 \times 0.98 \mathrm{~mm}^{3}\right)$ and b) an analog microphone $\left(3.35 \times 2.5 \times 0.98 \mathrm{~mm}^{3}\right)$

Analog microphones have a smaller footprint and fewer connecting pads. Two additional pads are necessary for digital microphones. More functions integrated in the ASIC chip result in a larger ASIC. These functions are shown in fig 3.

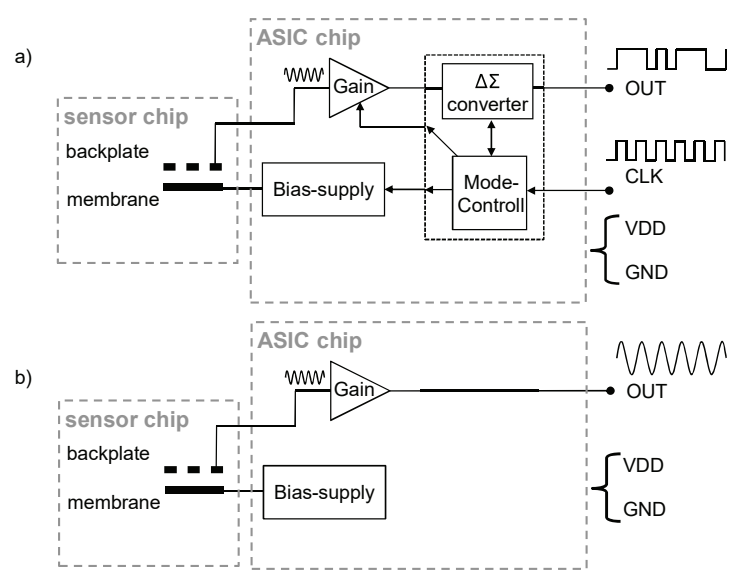

Fig. 3: Block diagram of a a) digital and an b) analog MEMS microphone

The sensor chip is a plate capacitor with a membrane and two perforated rigid backplates. The membrane is charged by a bias-supply from the ASIC. An incoming sound wave causes deflections of the membrane. The result is a change of the capacity. The ASIC detects the voltage change and amplifies it. An analog microphone transmits this signal to the output. A digital microphone uses a $\Sigma \Delta$ converter (fig. 4) and transforms the signal into a digital PDM (pulse density modulated) signal.

\section{Principal Function of a digital MEMS microphone}

In addition to the power supply (VDD) the digital microphones need an external clock signal. With the frequency of this signal, different modes can be selected. The modes differ in their power consumption and some electro-acoustic parameters.

This allows using the microphone's mode with the best acoustic parameters and the highest power consumption only when records with high quality are needed. This mode is called high mode. To save energy a low mode with lower power consumption and lower SNR is available. The L/R pin allows signal routing of the OUT data of two microphones only via one cable by defining the clock edge (falling or rising) at which the data of a microphone is sent.

\section{$\Delta \Sigma$-converter}

The $\Sigma \Delta$-converter translates the analog signal $X(s)$ from the sensor chip into a PDM output signal $\mathrm{Y}(\mathrm{s})$ consisting of ones and zeros. A simplified block diagram of such a first order $\Sigma \Delta$-converter is shown in fig. 4 in the Laplace s-domain. 
$N(s)$ : quantization noise

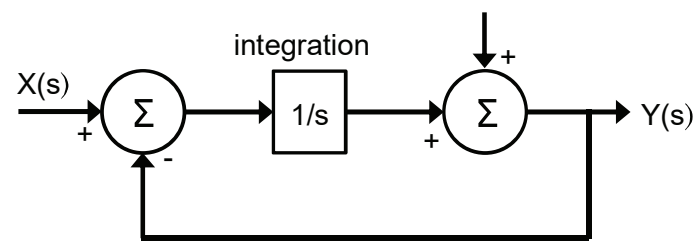

Fig. 4: Block diagram of a first order $\Sigma \Delta$ converter in the s-domain [9]

The output signal $Y(s)$ can be described with equation 2 by using the complex variable $s$.

$$
Y(s)=\frac{1}{s+1} X(s)+\frac{s}{s+1} N(s)
$$

with $s=\mathrm{j} \cdot 2 \cdot \pi \cdot \mathrm{f}$

$Y(s)$ is the sum of a signal transfer function and a noise transfer function. The signal transfer function in equation 3 in the s-domain has the characteristic of a low-pass filter [9] when $\mathrm{N}(\mathrm{s})=0$.

$$
\left|\frac{Y(s)}{X(s)}\right|=\left|\frac{1}{s+1}\right|
$$

For $\mathrm{f} \rightarrow 0$ the signal transfer function converges to 1 . The noise transfer function in equation 4 shows a high-pass behavior when $\mathrm{X}(\mathrm{s})=0$. [9]

$$
\left|\frac{Y(s)}{N(s)}\right|=\left|\frac{s}{s+1}\right|
$$

For $f \rightarrow \infty$ the output is dominated by the quantization noise. This is called noise shaping or shifting. This behavior can be seen in fig. 7 . If the input signal is oversampled, the quantization noise is shifted at high frequencies and the noise density in the bandwidth of interest significantly decreases. This shows why the $\Sigma \Delta$-converter is well suited for these audio applications. The quantization noise is shifted to frequencies that are higher than the audio frequencies. The order of the $\Sigma \Delta$-converter defines how strong this effect is. The typical order can be taken from the datasheet of a digital MEMS microphone and lies mostly at 4 . The main task of the $\Sigma \Delta$ converter is to transform the analog input voltage into +1 and 0 at the output. Fig. 5 shows a simplified PDM output signal and the input signal in the time domain.

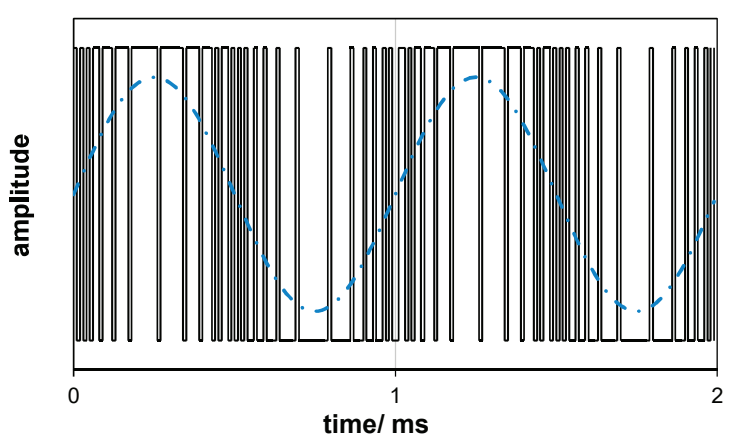

Fig. 5: Input and Output signal of a $\Sigma \Delta$ converter

Each clock cycle leads to a new output sample. When the input signal has a large absolute slope factor, you can see rapid changes between +1 and 0 . A small absolute slope factor in the analog signal results in a large amount of ones or zeros during that time.

\section{Characterization methods}

Two methods to calculate the characteristics of a digital MEMS microphone with a PDM output signal were investigated. The first method is based on a spectral analysis of the PDM signal. The main parts of the second method are a transformation to an analog signal and a down sampling process before an analytical calculation. These two ways are shown in figure 6 .



Fig. 6: $\quad$ Two methods to calculate the electroacoustic parameters from the digital PDM signal

All signal measurements were done with an acoustic chamber, which is explained in detail in [10].

\section{Method I: Spectrum Analysis of the PDM signal}

The spectrum of the PDM output signal is shown in figure 7 . The microphone was excited with a sound pressure of $1 \mathrm{~Pa}$ at $1 \mathrm{kHz}$. The spectrum calculation was scaled by the factor $2^{0.5}$. This is necessary because in the case of digital MEMS microphones only the peak values have to be considered by the sensitivity calculation. This means that if a sinusoidal 
curve with a full-scale amplitude is fed into the calculation, the spectrum peaks at $0 \mathrm{dBFS}$.



Fig. 7: Spectrum of the output signal of a digital MEMS microphone in high mode exited with a $1 \mathrm{kHz}$ signal with $1 \mathrm{~Pa}$.

The spectrum shows that the $\Sigma \Delta$-converter properly works because the noise is shifted out of the audio band $(20 \mathrm{~Hz}-22 \mathrm{kHz})$ to higher frequencies. The peak at $1 \mathrm{kHz}$ results from the incoming sound wave. With its height the sensitivity can be calculated. The unit of sensitivity is dBFS. This is an indicator of how much of the $\Sigma \Delta$-converter is modulated, calculated in dB. A fully modulated $\Sigma \Delta$ converter would lead to a sensitivity of $0 \mathrm{dBFS}$. A sensitivity of $-26 \mathrm{dBFS}$ shows that the signal peaks $26 \mathrm{~dB}$ below the full-scale value of $0 \mathrm{dBFS}$. Harmonics from the fundamental frequency are also visible. With the heights of the harmonics, the THD can be calculated with equation 1. A typical value of $0.2 \%$ is reached with the microphones used for this test. Another very important parameter of MEMS microphones is the noise. It can also be calculated from the spectrum. An A-weighted filter is applied on the time signal. After that a spectrum is calculated, which can be seen in figure 8 .

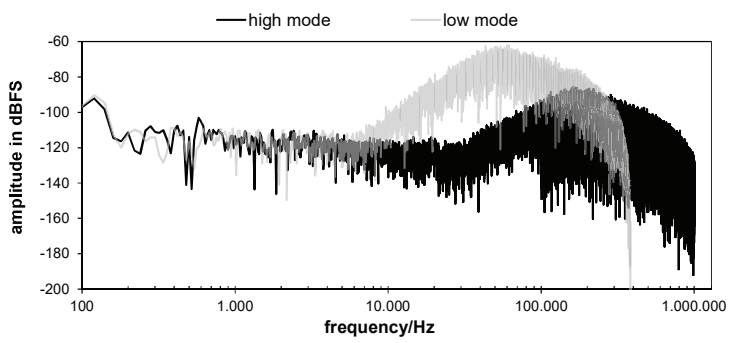

Fig. 8: A-weighted spectrum of the output signal at noise conditions of a digital MEMS microphone in high mode $\left(f_{\text {clock }}=2.4 \mathrm{MHz}\right)$ and low mode $\left(f_{\text {clock }}=768 \mathrm{kHz}\right)$

The spectrums show different behaviors. The noise shifting in high mode is well suited because the quantization peak is shifted out of the audio frequency band. In low mode the noise level begins to rise at $7 \mathrm{kHz}$. This is part of the audio band so it will be audible and reduces the acoustic performance.
In some datasheets a bandpass filter from $20 \mathrm{~Hz}$ to $7-8 \mathrm{kHz}$ is applied to calculate the noise or SNR value. This shows that in low mode a deteriorated SNR is to be expected. That is why low mode is not useful when records with high quality are to be taken. But the power consumption is lower than in high mode. Typical values for the SNR are between 64 and $65 \mathrm{~dB}(\mathrm{~A})$.

The noise value is calculated by summing up all squared amplitudes over a frequency span. After root extraction a window correction is made. The SNR is the difference between the sensitivity and the noise value.

The spectral analysis has a big disadvantage. To reach a high frequency resolution $\Delta \mathrm{f}$ a long period of time has to be recorded and this means a huge amount of data points N. A high frequency resolution is especially required to determine the sensitivity for low acoustic frequencies. If the signal frequency has no explicit frequency point in the spectrum, an amplitude defect can be shown. Equation 5 shows how the frequency resolution $\Delta f$ can be calculated.

$$
\Delta f / H z=\frac{f_{C L O C K} / H z}{N}
$$

For example, to reach a resolution of $5 \mathrm{~Hz}$ in the spectrum the signal has to be recorded for $200 \mathrm{~ms}$ at a sampling frequency of $2.4 \mathrm{MHz}$. More data points would lead to a higher calculating time for the spectrum. A long testing time results in higher costs and a lower throughput. In the next section this result is compared with the second method, to find out if shorter calculating times are possible.

\section{Method II: Low-pass-filtering and down- sampling}

The digital PDM output signal of the microphone is sampled by the clock frequency. After that this highly oversampled signal can be transformed into the analog domain by applying a low-pass filter. The rise times of such different filters are shown in figure 9. 




Fig. 9: $\quad$ Rise times of different low-pass filters

A higher order of the filter leads to a longer rise time. A bessel filter has a much smaller overshoot than a butterworth filter. In additon a bessel filter has a shorter rise time. The fact that the bessel filter has a larger transition frequency area was the reason to use a butterworth filter for the analog conversion. The large transition frequency area is shown in fig. 10.

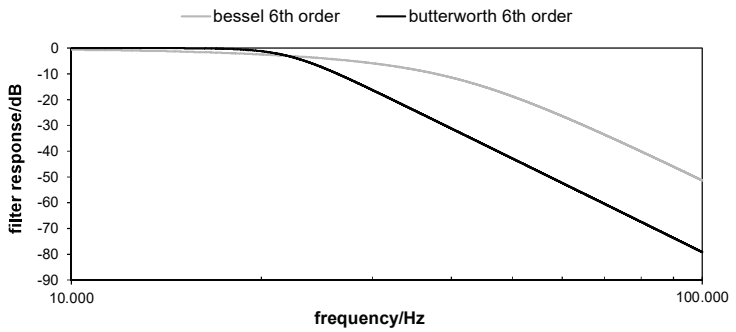

Fig. 10: Frequency response of a $6^{\text {th }}$ order bessel and butterworth low-pass filter

Both filters have a cut-off frequency of $22 \mathrm{kHz}$ and an order of six. The butterworth filter shows a higher damping above $22 \mathrm{kHz}$ than the bessel filter. The 350 sampling points during the rise of the filter must be neglected. This corresponds to an interval of approx. $150 \mu \mathrm{s}$ in the high mode and $500 \mu \mathrm{s}$ in the low mode. The order has to be chosen to reduce the share of high frequency noise in the analog signal (fig. 11).

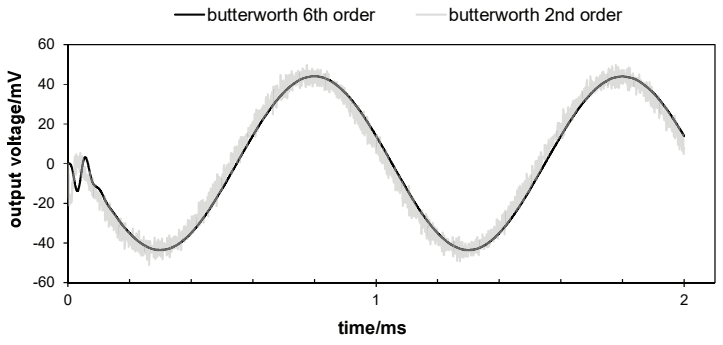

Fig. 11: Output signal of a butterworth filter with an order of 2 and 6

An order of 6 and a cut-off frequency of $22 \mathrm{kHz}$ can clear out high frequency noise outside the audio frequency band. To reduce the number of data points the analog signal has to be decimated to an audio bandwidth of $24 \mathrm{kHz}$. This means a reduction of the amount of points by $50\left(f_{\text {CLOCK }}=2.4 \mathrm{MHz}\right)$ in high mode and 16 $\left(\mathrm{f}_{\mathrm{CLOCK}}=768 \mathrm{kHz}\right)$ in low mode. The reduction is done by calculating an average value over 50 or 16 input sampling data points.

The sensitivity can then be calculated with equation 6 . This is an amplitude calculating routine in the time domain by summing up all squared data points before dividing by the number of points.

sensitivity $/ d B F S=$

$20 \cdot \log \left(\sqrt{\frac{2 \cdot\left(x_{1}^{2}+x_{2}^{1}+\ldots+x_{N}^{2}\right)}{N}}\right)$

The noise value has to be calculated by a spectrum analysis of the analog and decimated signal. The noise spectrum in high mode is shown in figure 12.

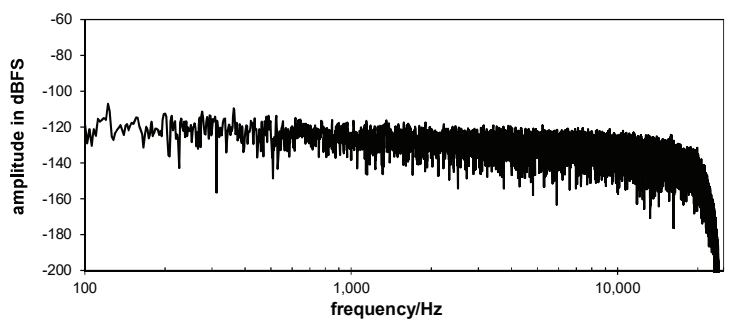

Fig. 12: A-weighted spectrum of the analog and decimated output signal at noise conditions

The evaluation of the analog signal shows that the same values for the electro-acoustic characteristics as with method I could be calculated. But there is still a difference in the time which has to be spent on calculating these characteristics with the digital PDM output signal.

\section{Comparison of the two methods}

The two methods described for the calculation of the characteristics of digital MEMS microphones need different amounts of time to calculate useful values. This is a result of the different number of measuring points in each method. The digital PDM output is not decimated therefore the number of measuring points is much higher than for the transformed analog signal which represents the same lime length. The time spent for calculating sensitivity for each method is shown in figure 13. 




Fig. 13: Time spent for calculating sensitivity for the two methods in high mode

Transformation into an analog signal followed by a decimation process needs less time for calculating than the spectral analysis of the PDM signal. This effect occurs when the signal length is longer than $30 \mathrm{~ms}$. For example, a $108 \mathrm{~ms}$ input signal needs $27 \mathrm{~ms}$ for calculating the noise with method I and only 16 ms by applying method II. Normally an input signal length from $40-100 \mathrm{~ms}$ is used to calculate the characteristics. The curve for method I only consists of a few points which are not connected. These points show that the number of measuring points, which are used for the calculation have values of $2^{n}$. With that number of points, the Fast Fourier Transformation (FFT) can be used. For other numbers of measurement points the very slow Discrete Fourier Transformation (DFT) is used. The calculating time for a DFT is not shown. Significantly longer times are necessary for the DFT. The times for noise calculation are shown in fig 14.

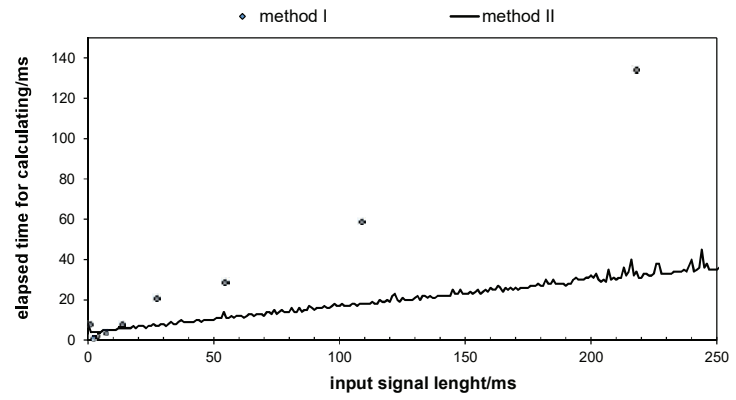

Fig. 14: Time spent for calculating noise for the two methods in high mode

For calculating noise the difference in the elapsed calculating time between method I and II is larger. For example a $108 \mathrm{~ms}$ input signal needs $50 \mathrm{~ms}$ for calculating the noise with method I and only $18 \mathrm{~ms}$ with method II. This also shows that sensitivity calculation is quicker than noise calculation.

\section{Conclusion}

Current trends suggest that in the future digital MEMS microphones will take a leading role in consumer audio applications. They show a good immunity against RF interference and have a high SNR. In this paper we described methods to measure the main parameters of digital microphones more easily and faster.

With the first method, a spectrum analysis of the PDM signal is applied to calculate sensitivity, THD and noise. The second method is more elaborate. The digital signal is converted into the analog domain. After that the analog waveform is highly oversampled. To avoid this large number of data points a decimation process is applied. With this signal and a bandwidth of $22 \mathrm{kHz}$ the characteristic parameters are calculated. The calculating of the signal in method II is faster compared to method I. If the number of measurement points is not given by $2^{n,}$ method $I$ is very slow. On average method II is 60 percent faster for noise calculation and $30 \%$ faster for sensitivity calculation.

\section{Literature}

[1] G. M. Sessler, Silicon microphones, J. Audio Eng. Soc. 44, 1996, pp. 16-22

[2] G. W. Elko, F. Pardo, D. Lopez, D. Bishop, P. Gammel, Capacitive MEMS mircrophones, Bell Labs Technical Journal 10, 2005, pp. 187-198, 10.1002/bltj.20113

[3] J. Lewis, Analog and Digital MEMS Microphone Design Considerations, Technical Article MS2472, February 2015

[4] A. Dehe, M. Wurzer, M. Füldner, U. Krumbein, The Infineon Silicon MEMS Microphone, Proceedings of AMA Conference, Nürnberg, Germany May 14-16, 2013, pp. 98-99, 10.5162/sensor2013/A4.3

[5] G. Feiertag, M. Winter, A. Leidl, Packaging of MEMS Microphones, Proceedings of Smart Sensors, Actuators and MEMS, Dresden, Germany, May 04, 2009, vol. 7362, $10.1117 / 12.821186$

[6] J. Bay, O. Hansen, S. Bouwstra, Micromachined double backplate differential capacitive microphone, Journal of Micromechanics and Microengineering, Vol 9 1999, pp 30-33

[7] P. Rombach, M. Müllenborn, U. Klein, K. Rasmussen, The first low voltage, low noise differential silicon microphone, technology development and measurement results, Proceedings of International Conference on Micro Electro Mechanical Systems, 2001, pp. 42-45, 10.1109/MEMSYS.2001.906474 
[8] D.T. Martin, K. Kardirvel, J. Liu, R.M. Fox, M. Sheplak, T. Nishida, Surface and Bulk Micromachined Dual Back-Plate Condenser Microphone, Proceedings of Micro Electro Mechanical Systems Conference, Jan 30-Feb 03, 2005, pp. 319-322,

10.1109/MEMSYS.2005.1453931

[9] S. Park, Principles of Sigma-Delta Modulation for Analog-to-Digital Converters, Motorola Application Note, 1996

[10] S. Walser, M. Loibl, G. Feiertag, C. Siegel, Fertigungsendtest für MEMS Mikrofone, Virtuelle Instrumente in der Praxis 2015, Begleitband zum 20. VIP-Kongress, pp. 302307; ISBN 978-3-8007-3669-0, 2015 